\title{
Generalization of the Beck-Cohen superstatistics
}

\author{
Denis Nikolaevich Sob’yanin* \\ Tamm Department of Theoretical Physics, \\ Lebedev Physical Institute, Russian Academy of Sciences, \\ Leninskii pr. 53, Moscow 119991, Russia
}

(Received 16 September 2011; Revised 24 October 2011; Published 28 November 2011)

\begin{abstract}
Generalized superstatistics, i.e., a "statistics of superstatistics," is proposed. A generalized superstatistical system comprises a set of superstatistical subsystems and represents a generalized hyperensemble. There exists a random control parameter that determines both the density of energy states and the distribution of the intensive parameter for each superstatistical subsystem, thereby forming the third, upper level of dynamics. Generalized superstatistics can be used for nonstationary nonequilibrium systems. The system in which a supercritical multitype age-dependent branching process takes place is an example of a nonstationary generalized superstatistical system. The theory is applied to pair production in a neutron star magnetosphere.
\end{abstract}

PACS numbers: 05.70.Ln, 05.40.-a, 02.50.Ey, 97.60.Jd

\section{INTRODUCTION}

A large variety of complex nonequilibrium systems exhibit spatiotemporally inhomogeneous dynamics. Such systems are often characterized by hierarchical structures of dynamics. The hierarchy can be formed by the decomposition of the system dynamics into different dynamics on different spatiotemporal scales, which are largely separated from each other. In this case, the statistical properties of the system can be effectively described by a superposition of several statistics, or a "superstatistics."

Superstatistics has been formulated in Ref. [1] to consider nonequilibrium systems with a stationary state and intensive parameter fluctuations. Some preliminary concepts have been anticipated earlier in Refs. 2-4]. Superstatistical systems are characterized by the existence of an intensive parameter $\beta$ that fluctuates on a much larger time scale than the typical relaxation time of the local dynamics. If a given system can be thought of as a collection of many small spatial cells, then the inverse temperature in a cell is typically taken as such a parameter. However, more general interpretations of the intensive parameter are possible. Sufficient time scale separation between two relevant dynamics within the complex system allows one to qualify superstatistics as a form of slow modulation [5].

Superstatistics is applicable to various complex systems. Its applications include, among others, cosmicray energy spectra and electron-positron pair annihilation [6, 7], the world line representations of Feynman propagators for spin- 0 and spin- $1 / 2$ particles [8], an extension of the random matrix theory covering systems with mixed regular-chaotic dynamics 9 -11], nonstationary dynamical processes with time-varying multiplicative noise exponents [12], Markovian systems without detailed balance [13], a mesoscopic approach to the problem of

\footnotetext{
* sobyanin@lpi.ru
}

Brownian motion [14], models of the metastatic cascade in cancerous systems [15], complex networks 16], ecosystems driven by hydroclimatic fluctuations [17], patternforming systems [18], solar flares [19], share price fluctuations [20 23], the statistics of train departure delays 24], wind velocity fluctuations [25], and many interesting applications in hydrodynamic turbulence [23, 26 30].

Let us suppose for a while that we have a complex nonequilibrium system described by superstatistics. One of the main problems is the determination of the distribution $f(\beta)$ of the intensive parameter $\beta$. It can be found using the maximum entropy principle 31. Moreover, $f(\beta)$ can be considered as a function of a set of additional control parameters $\left\{\lambda_{i}\right\}, f=f\left(\beta,\left\{\lambda_{i}\right\}\right)$ [32]. These parameters emerge as Lagrange multipliers determined by maximizing the Boltzmann-Gibbs-Shannon entropy of $f\left(\beta,\left\{\lambda_{i}\right\}\right)$ under certain constraints. Let us revert to the parameter $\beta$. In each cell of the superstatistical system, we have the Gibbs canonical distribution $\rho_{G}(E \mid \beta)=e^{-\beta E} / Z(\beta)$, where $Z(\beta)$ is the partition function. This distribution can be obtained by maximizing the Boltzmann-Gibbs-Shannon entropy associated with a normalized distribution $\rho(E \mid \beta)$, where $\beta$ is the Lagrange multiplier corresponding to the mean energy constraint. We see that, in general, both $\beta$ and $\left\{\lambda_{i}\right\}$ have a similar nature. However, in superstatistics, $\beta$ fluctuates, but the control parameters are constant.

We can pose the following question: What statistics appears in the case of fluctuating control parameters? The aim of this paper is to develop the generalization of the Beck-Cohen superstatistics that allows one to properly consider such fluctuations. These form a separate, upper level of dynamics, thus leading to multiscale superstatistics. The possible existence of such superstatistics has been presumed in Ref. [31].

This paper is organized as follows. In Sec. II generalized superstatistics is developed. In Sec. III supercritical multitype age-dependent branching processes are considered as an example of generalized superstatistical systems. In Sec. IV generalized superstatistics is applied to 
electron-positron pair production in a neutron star magnetosphere and the particle energy distribution is calculated. In Sec. V the main results are summarized.

\section{GENERALIZED SUPERSTATISTICS}

Let us consider a nonequilibrium system that consists of a set of nonequilibrium subsystems described by their superstatistics. This system can be referred to as a "generalized superstatistical system." Let us define a random variable $\xi$ that determines the properties of each nonequilibrium superstatistical subsystem. The role of $\xi$ in determining the properties of the subsystems is similar to that of $\beta$ in determining the properties of the cells that constitute a superstatistical subsystem. In other words, $\xi$ is a control parameter, which determines the form of the superstatistical distribution for each subsystem. Here, we do not restrict ourselves to considering $\xi$ as a scalar random variable. In principle, $\xi$ is allowed to be a multidimensional random vector. The distribution of $\xi$ is characterized by a probability density $c(\xi)$, which is normalized, $\int c(\xi) d \xi=1$.

We can look at the generalized superstatistical system using the hyperensemble approach [33]. Developed in the context of nonequilibrium systems, this approach is consistent with the standard theory of equilibrium statistical physics [34]. Superstatistics can be considered as the theory of hyperensembles [35]. Each superstatistical subsystem of the system represents a hyperensemble, i.e., a mixture of canonical ensembles. In turn, the system as a whole can be described by a mixture of the hyperensembles corresponding to the subsystems. In other words, the generalized superstatistical system represents a "generalized hyperensemble," i.e., an ensemble of hyperensembles.

There are three levels of dynamics in the system under consideration: The first level is the level of fast dynamics in a cell. The second level is the level of superstatistical dynamics in a subsystem. The existence of an additional dynamics described by $\xi$ forms the third level in the hierarchy of dynamics and allows us to refer to the statistics of the generalized superstatistical system as a "generalized superstatistics," i.e., a statistics of superstatistics.

Consider a superstatistical subsystem of the generalized superstatistical system. Let $\Gamma(E \mid \xi)$ be a nondecreasing function representing the number of states with energy less than $E$. The random variable $\xi$ determines the density of energy states for the subsystem,

$$
g(E \mid \xi)=\frac{\partial \Gamma(E \mid \xi)}{\partial E} .
$$

The inclusion of the density of states in the superstatistical description is significant [36]. When writing $d \Gamma(E \mid \xi)$ in integrals, we will imply integration over $E$ so that $d \Gamma(E \mid \xi)=g(E \mid \xi) d E$. The Gibbs canonical distribution for each cell of the subsystem is

$$
\rho_{G}(E \mid \beta, \xi)=\frac{e^{-\beta E}}{Z(\beta \mid \xi)},
$$

where

$$
Z(\beta \mid \xi)=\int e^{-\beta E} d \Gamma(E \mid \xi)
$$

is the partition function. By analogy with the approach used in Ref. [32], we do not include the density of states in the definition of the energy distribution (1); therefore, the normalization condition is $\int \rho_{G}(E \mid \beta, \xi) d \Gamma(E \mid \xi)=1$. We also assume that $\xi$ determines the distribution $f(\beta \mid \xi)$ of the intensive parameter $\beta$. This distribution is normalized, $\int f(\beta \mid \xi) d \beta=1$. Note that $g(E \mid \xi)$ and $f(\beta \mid \xi)$ are not necessarily statistically dependent variables. These will change independently if we choose $\xi=\left(\xi_{1}, \xi_{2}\right)$, with $\xi_{1}$ and $\xi_{2}$ being independent random variables, and set $g(E \mid \xi)=g\left(E \mid \xi_{1}\right)$ and $f(\beta \mid \xi)=f\left(\beta \mid \xi_{2}\right)$.

The superstatistical distribution for the subsystem is given by

$$
\rho(E \mid \xi)=\int \rho_{G}(E \mid \beta, \xi) f(\beta \mid \xi) d \beta,
$$

with the normalization condition being $\int \rho(E \mid \xi) d \Gamma(E \mid \xi)=1$. If $\xi$ is a nonrandom vector, then this distribution reduces to the ordinary superstatistical distribution [1]. By averaging Eq. (2) over the fluctuating $\xi$, we immediately obtain the generalized superstatistical distribution

$$
\sigma(E)=\int \rho(E \mid \xi) g(E \mid \xi) c(\xi) d \xi,
$$

which is normalized, $\int \sigma(E) d E=1$.

\section{BRANCHING PROCESSES}

Now the following question arises: Do there exist any nonequilibrium systems, whether stationary or not, that can be described by generalized superstatistics?

Let us consider a many-particle system composed of particles of $n$ types. Each type- $i$ particle, which will also be denoted by $T_{i}, 1 \leqslant i \leqslant n$, has a random lifetime with a probability distribution function $G_{i}(\tau)$. In other words, $G_{i}(\tau)$ is the probability that the lifetime of a given type- $i$ particle does not exceed $\tau$. At the end of its life the particle decays into a random number of particles of several types. Specifically, at the moment of its decay the particle produces $\omega_{j} \geqslant 0$ type- $j$ particles of age zero, $1 \leqslant j \leqslant n$ :

$$
T_{i} \rightarrow \sum_{j=1}^{n} \omega_{j} T_{j} .
$$

Thus we have a multitype age-dependent branching process, the so-called multitype Sevast'yanov process [37]. 
The transformation (4) is described by the generating function

$$
h_{i}(\tau, x)=\sum_{\omega} p_{i}^{\omega}(\tau) x_{1}^{\omega_{1}} \cdots x_{n}^{\omega_{n}},
$$

where $p_{i}^{\omega}(\tau)$ is the conditional probability of the transformation (41) given that the particle decays at age $\tau$, $\omega=\left(\omega_{1}, \ldots, \omega_{n}\right)$ is an $n$-dimensional vector with nonnegative integer components $\omega_{j}$, and $x=\left(x_{1}, \ldots, x_{n}\right)$ is an $n$-dimensional vector with complex components $x_{j}$ such that $\left|x_{j}\right| \leqslant 1$. If $x_{1}=\cdots=x_{n}=1$, we will write $x=1$. The normalization condition reads $h_{i}(\tau, 1)=1$.

The mean number of type- $j$ particles that appear upon the decay of a type- $i$ particle is given by

$$
A_{i j}=\int_{0}^{\infty} a_{i j}(\tau) d G_{i}(\tau)
$$

where

$$
a_{i j}(\tau)=\left.\frac{\partial h_{i}(\tau, x)}{\partial x_{j}}\right|_{x=1}
$$

is the same mean given that the particle decays at age $\tau$. We will assume that the $n \times n$ matrix $A=\left\|A_{i j}\right\|$ with components $0 \leqslant A_{i j}<\infty$ is irreducible, or indecomposable, i.e., the index set $\{1, \ldots, n\}$ cannot be divided into two disjoint nonempty sets $S_{1}$ and $S_{2}$ such that $A_{i j}=0$ for all $i \in S_{1}$ and all $j \in S_{2}$ (see, e.g., Ref. [38] for details). Moreover, we will also assume that the Perron root of $A$, i.e., the maximum positive real eigenvalue of $A$, is greater than one. Thus we deal with the indecomposable supercritical branching process [39]. Physically, this means that, first, a particle of a given type potentially has descendants, either direct or distant, of any type and, second, the number of particles in the system, on average, progressively increases.

We see that the whole system is composed of $n$ subsystems, the $i$ th subsystem comprising type- $i$ particles. Obviously, the subsystems interact with each other in the sense that the decay of a particle in one subsystem leads to the creation of particles in other subsystems. The number of particles both in the whole system and in the subsystems is not constant and changes with time. Thus we have a nonstationary nonequilibrium situation.

Let $\mu(t)=\left[\mu_{1}(t), \ldots, \mu_{n}(t)\right]$ be an $n$-dimensional vector with non-negative integer components. Each $\mu_{j}(t)$ is a random variable that yields the number of particles in the $j$ th subsystem at time $t$. The age-dependent branching process is characterized by a set of generating functions

$$
F_{i}(t, x)=\sum_{\omega} P_{i}^{\omega}(t) x_{1}^{\omega_{1}} \cdots x_{n}^{\omega_{n}}
$$

where $P_{i}^{\omega}(t)$ is the conditional probability that $\mu(t)=\omega$ given that there is one particle in the $i$ th subsystem and none in the other subsystems at time zero. The generating functions $F_{i}(t, x)$ (6) $), 1 \leqslant i \leqslant n$, satisfy a system of nonlinear integral equations [37]. Essential for us here is the probabilistic nature of the number of particles both in the whole system and in each subsystem. In general, we cannot predict a priori what the number of particles is in each subsystem at a given time, but instead we can focus on the average behavior of the generalized hyperensemble.

The mean number of particles in the $j$ th subsystem at time $t$ given that the branching process has started with one type- $i$ particle at time zero is

$$
A_{i j}(t)=\left.\frac{\partial F_{i}(t, x)}{\partial x_{j}}\right|_{x=1} .
$$

To analyze the long-run behavior of these means, let us define the Laplace-Stieltjes transforms

$$
L_{i j}(\alpha)=\int_{0}^{\infty} e^{-\alpha \tau} d G_{i j}(\tau),
$$

where

$$
G_{i j}(t)=\int_{0}^{t} a_{i j}(\tau) d G_{i}(\tau) .
$$

Let $\lambda(\alpha)$ be the Perron root of the $n \times n$ matrix $L(\alpha)=$ $\left\|L_{i j}(\alpha)\right\|$. Choose an $\alpha$ such that $\lambda(\alpha)=1$ and define an $n$-dimensional left eigenvector $v=\left(v_{1}, \ldots, v_{n}\right)$ of $L(\alpha)$ with positive real components $v_{j}$ that satisfy

$$
v_{j}=\sum_{i=1}^{n} v_{i} L_{i j}(\alpha)
$$

Then the asymptotic behavior of the mean numbers of particles is expressed as

$$
A_{i j}(t) \sim C_{i} v_{j} w_{j} e^{\alpha t}, \quad t \rightarrow \infty,
$$

where

$$
w_{j}=\int_{0}^{\infty} e^{-\alpha \tau}\left[1-G_{j}(\tau)\right] d \tau
$$

and $C_{i}$ is a positive constant [40]. Interestingly, $A_{i j}(t)$ behaves in a similar way for each subsystem. Since we consider the supercritical case, $\alpha$ always exists and is positive. Therefore, the mean number of particles in each subsystem increases exponentially. Note that the existence of a positive $\alpha$ is equivalent to the condition that the Perron root of $A$ is greater than one 39, 40].

In the long-term run, the limiting probability that a given particle belongs to the $i$ th subsystem can be written as

$$
\pi_{i}=\frac{v_{i} w_{i}}{\sum_{j=1}^{n} v_{j} w_{j}} .
$$

Note that $\pi_{i}$ is independent of the type of the primary particle with which the branching process has started. Moreover, nonstationary though the situation is, the limiting probability is stationary. 
The limiting age distribution for the $i$ th subsystem is

$$
L_{i}(\tau)=\frac{\int_{0}^{\tau} e^{-\alpha u}\left[1-G_{i}(u)\right] d u}{\int_{0}^{\infty} e^{-\alpha u}\left[1-G_{i}(u)\right] d u} .
$$

It is found by dividing the $i$ th subsystem into two subsystems, the first comprising the type- $i$ particles that decay at age $u \leqslant \tau$ and the second comprising the rest, and applying the technique described above to the new system. Equation (11) yields the probability that a randomly chosen type- $i$ particle decays at age $u \leqslant \tau$. In the case of a single type of particles, $L_{i}(\tau)$ reduces to the limiting age distribution for the one-dimensional supercritical Sevast'yanov process [41], which coincides with the classical age distribution for the one-dimensional supercritical Bellman-Harris process when the probability $p_{i}^{\omega}(\tau)$ is independent of $\tau, p_{i}^{\omega}(\tau)=p_{i}^{\omega}$ [42, 43].

Now we can calculate the particle energy distribution. Consider a type- $i$ particle of age $\tau$. In general, its energy can be considered as a random variable characterized by a conditional probability density $w_{i}(E \mid \tau)$. More specifically, the probability that the energy of a type- $i$ particle of age $\tau$ lies in a small interval $d E$ around $E$ is $w_{i}(E \mid \tau) d \Gamma_{i}(E)$, where $\Gamma_{i}(E)$ is the number of energy states with energy less than $E$. The normalization condition is $\int w_{i}(E \mid \tau) d \Gamma_{i}(E)=1$. The energy probability density for the $i$ th subsystem becomes

$$
\rho_{i}(E)=\int_{0}^{\infty} w_{i}(E \mid \tau) d L_{i}(\tau)
$$

with the normalization condition being $\int \rho_{i}(E) d \Gamma_{i}(E)=$ 1 .

The described system can be considered as a generalized superstatistical system. The control parameter $\xi$ is a discrete random variable that takes on values $\{1, \ldots, n\}$ and yields the number of the subsystem to which a randomly chosen particle belongs. In other words, $\xi$ corresponds to the particle type. It has the discrete probability distribution $\left\{\pi_{1}, \ldots, \pi_{n}\right\}$, where $\pi_{i}$ is given by Eq. (10). It remains to find the distribution $f_{i}(\beta)$ of the fluctuating parameter $\beta$ for each subsystem. Let

$$
g(s)=\mathfrak{L}[f(x)](s)=\int_{0}^{\infty} e^{-s x} f(x) d x
$$

be the Laplace transform of a function $f(x)$, with $f(x)=$ $\mathfrak{L}^{-1}[g(s)](x)$ being the corresponding inverse Laplace transform. The energy distribution for the $i$ th subsystem can be expressed as

$$
\rho_{i}(E)=\mathfrak{L}\left[\frac{f_{i}(\beta)}{Z_{i}(\beta)}\right](E),
$$

where $Z_{i}(\beta)$ is the partition function. Then

$$
f_{i}(\beta)=Z_{i}(\beta) \mathfrak{L}^{-1}\left[\rho_{i}(E)\right](\beta) .
$$

\section{AN EXAMPLE: PAIR PRODUCTION IN A NEUTRON STAR MAGNETOSPHERE}

New nonstationary cosmic radio sources associated with neutron stars, viz., intermittent pulsars 44] and rotating radio transients (RRATs) [45], have been discovered recently. The essential feature of these sources is their long "silence," when we do not observe any radio emission from them. Since an electron-positron plasma outflowing from the magnetosphere of a neutron star is responsible for the observable radio emission, the plasma generation can be switched off for some time. In this case, the absorption of a high-energy photon in the inner neutron star magnetosphere triggers nonstationary cascade pair production [46], which, in turn, results in the formation of a "lightning" 47]. Such lightnings can manifest themselves as radio bursts from RRATs [48]. In a lightning, we deal with an ultrarelativistic electronpositron plasma. The properties of the emission from electrons and positrons are determined by their energies. Therefore, it is important to find the energy distribution of particles. This can be done using generalized superstatistics.

Let us characterize the energy of a charged particle, either an electron or a positron, by its Lorentz factor $\gamma(\tau)$. The particle is efficiently accelerated by a longitudinal electric field $E_{\|}$so that $\gamma(\tau)$ eventually reaches a stationary value $\gamma_{0}$, which is $\sim 10^{8}$ in a vacuum neutron star magnetosphere [49]. The electron and positron of each produced pair, though ultrarelativistic, initially have Lorentz factors much less than $\gamma_{0}$. At the initial stage of acceleration $\gamma(\tau)$ increases linearly with time, $\gamma(\tau) \approx E_{\|} \tau$. Here, we use a dimensionless system of units (see, e.g., Ref. [48]). By contrast, when $t$ approaches $\tau_{0}=\gamma_{0} / E_{\|}$, the radiation forces come to the fore, and a need arises to use the Dirac-Lorentz equation to consider the particle dynamics properly 49 51].

Thus, we can define two types of particles: A type-1 particle can be efficiently accelerated by the electric field since the radiation friction is negligible. A type- 2 particle, in contrast to a type- 1 particle, is not accelerated by the electric field because of the electrodynamic selfaction effects and has the constant Lorentz factor $\gamma_{0}$. In the former case the particle does not efficiently produce secondary pairs, but in the latter case it does at a rate $Q$ [46]. Note that the plasma generation, along with the accompanying radio emission, is not suppressed even in ultrahigh magnetar magnetic fields [52, 53]. The Lorentz factors of type- 1 and type- 2 particles as functions of their ages become

$$
\begin{array}{ll}
\gamma_{1}(\tau)=E_{\|} \tau, & 0 \leqslant \tau<\tau_{0}, \\
\gamma_{2}(\tau)=\gamma_{0}, & 0 \leqslant \tau<\infty .
\end{array}
$$

The particles of each produced pair, though moving independently of each other, can conveniently be considered as a whole, and type- 1 and type- 2 pairs, which will be denoted by $T_{1}$ and $T_{2}$, respectively, are defined by analogy with individual particles. 
Now we can write the following transformations:

$$
\begin{aligned}
& T_{1} \rightarrow T_{2}, \\
& T_{2} \rightarrow T_{1}+T_{2} .
\end{aligned}
$$

The generating functions are

$$
\begin{aligned}
& h_{1}(\tau, x)=x_{2}, \\
& h_{2}(\tau, x)=x_{1} x_{2},
\end{aligned}
$$

where $x=\left(x_{1}, x_{2}\right)$. The $2 \times 2$ matrix $a=\left\|a_{i j}\right\|$ becomes $\left(\begin{array}{ll}0 & 1 \\ 1 & 1\end{array}\right)$. The lifetime distribution functions are

$$
\begin{aligned}
& G_{1}(\tau)=\theta\left(\tau-\tau_{0}\right), \\
& G_{2}(\tau)=1-e^{-2 Q \tau},
\end{aligned}
$$

where $\theta(x)$ is the Heaviside function. The matrix $A$ defined by Eq. (5) coincides with $a$ and is indecomposable since its off-diagonal elements are positive. The Perron root of $A$ is equal to $(1+\sqrt{5}) / 2$ and greater than 1 . Therefore, we deal with the indecomposable supercritical two-type branching process.

Now we should find a proper value of $\alpha$ as described after Eq. (7). Interestingly, we can do this without directly finding the Perron root of $L(\alpha)$. We have recently shown [46] that pair production in the system under consideration is asymptotically described by the equation

$$
\frac{d N(t)}{d t}=2 Q^{\mathrm{eff}} N(t)
$$

where $N(t)$ is the number of electron-positron pairs at time $t, Q^{\text {eff }}=N_{\tau_{0}}^{\text {eff }} / 2 \tau_{0}$ is the effective pair production rate, and $N_{\tau_{0}}^{\text {eff }}$ satisfies

$$
N_{\tau_{0}}^{\mathrm{eff}}=\ln N_{\tau_{0}}-\ln N_{\tau_{0}}^{\mathrm{eff}},
$$

where $N_{\tau_{0}}=2 Q \tau_{0}$ is the number of particles created by a fully accelerated particle in time $\tau_{0}$. Note that $N_{\tau_{0}}^{\text {eff }}$ can be equivalently expressed through the Lambert function $W(x)$ (see, e.g., Ref. [54] for its definition) as

$$
N_{\tau_{0}}^{\mathrm{eff}}=W\left(N_{\tau_{0}}\right)
$$

and hence is positive since $W(x)$ is positive for any positive $x$. From Eq. (15) it follows that the total number of electron-positron pairs in the system increases exponentially. Comparing Eq. (15) to Eq. (8) yields

$$
\alpha=2 Q^{\mathrm{eff}}
$$

which is positive, as it must be in the supercritical case.

The energy of any type- 1 particle is less than $\gamma_{0}$, while that of any type- 2 particle is $\gamma_{0}$. Therefore, it is natural to choose the following density of states for the subsystems:

$$
\begin{aligned}
& g_{1}(\gamma)=1-\theta\left(\gamma-\gamma_{0}\right), \\
& g_{2}(\gamma)=\delta\left(\gamma-\gamma_{0}\right),
\end{aligned}
$$

where $\delta(x)$ is the delta function. We see from Eq. (13) that the energies of particles are nonrandom functions of their ages, hence the conditional energy distributions have the form

$$
\begin{aligned}
& w_{1}(\gamma \mid \tau)=\delta\left[\gamma-\gamma_{1}(\tau)\right], \\
& w_{2}(\gamma \mid \tau)=1
\end{aligned}
$$

Using Eqs. (11), (12), (14), and (18), we obtain the energy distributions for the subsystems:

$$
\begin{aligned}
& \rho_{1}(\gamma)=\frac{\alpha}{E_{\|}} \frac{e^{-\alpha \gamma / E_{\|}}}{1-e^{-\alpha \tau_{0}}}, \\
& \rho_{2}(\gamma)=1 .
\end{aligned}
$$

The corresponding intensive parameter distributions are

$$
\begin{aligned}
& f_{1}(\beta)=\delta\left(\beta-\frac{\alpha}{E_{\|}}\right), \\
& f_{2}(\beta)=\delta(\beta) .
\end{aligned}
$$

Let us find the probability distribution of the random control parameter $\xi$ that corresponds to the type of a randomly chosen particle. From Eqs. (9) and (14) we have

$$
\begin{aligned}
& w_{1}=\frac{1-e^{-\alpha \tau_{0}}}{\alpha}, \\
& w_{2}=\frac{1}{\alpha+2 Q} .
\end{aligned}
$$

The components of the left eigenvector $v=\left(v_{1}, v_{2}\right)$ of

$$
L(\alpha)=\left(\begin{array}{cc}
0 & e^{-\alpha \tau_{0}} \\
\left(1+\frac{\alpha}{2 Q}\right)^{-1} & \left(1+\frac{\alpha}{2 Q}\right)^{-1}
\end{array}\right)
$$

can be chosen as follows:

$$
\begin{aligned}
& v_{1}=1, \\
& v_{2}=1+\frac{\alpha}{2 Q} .
\end{aligned}
$$

Using Eqs. (10), (16), (20), and (21), we obtain the probability $\pi_{\xi}$ that a randomly chosen particle is of type $\xi$, $\xi=1,2$ :

$$
\begin{aligned}
& \pi_{1}=1-\frac{\alpha}{2 Q} \\
& \pi_{2}=\frac{\alpha}{2 Q} .
\end{aligned}
$$

Note that $\pi_{2}$ may be interpreted as the probability that the particle significantly contributes to pair production.

Finally, Eqs. (3), (16), (17), (19), and (22) allow us to obtain the generalized superstatistical distribution

$$
\sigma(\gamma)=\frac{\alpha}{2 Q} \delta\left(\gamma-\gamma_{0}\right)+\left[1-\theta\left(\gamma-\gamma_{0}\right)\right] \frac{\alpha}{E_{\|}} e^{-\alpha \gamma / E_{\|}}
$$

which represents the energy distribution of ultrarelativistic electrons and positrons.

The particle energy distribution in the neutron star magnetosphere cannot be observed directly. Moreover, 
it is unclear how to infer the particle energy distribution for RRATs from currently available observational data. On the one hand, it is difficult to detect radio emission from RRATs because of its sporadic nature, and one has to carry out single-pulse searches, which require significant radio telescope resources with long observation times [55, [56]. On the other hand, it is unclear how the form of the distribution correlates with the properties of radio emission. Although the parameters of an electronpositron plasma generated in the RRAT magnetosphere imply the possibility of the efficient generation of radio emission [47, 48], the detailed mechanism is unknown at the moment. Therefore, the distribution (23) is a theoretical prediction.

\section{CONCLUSION}

I have developed generalized superstatistics, i.e., a statistics of superstatistics. Based on the concept of fluctuating control parameters, it is defined for a generalized superstatistical system, which consists of a set of nonequilibrium superstatistical subsystems. Such a system can be considered as a generalized hyperensemble, i.e., an ensemble of hyperensembles. A fluctuating control parameter, which may be a multidimensional random vector, determines the form of the superstatistical distribution for each subsystem. In general, it determines not only the distribution of the intensive parameter but also the density of energy states. In a generalized superstatistical system, there appears the third, upper level in the hierarchy of dynamics besides two levels that exist in each superstatistical subsystem. Interestingly, generalized superstatistics can be applied to nonstationary nonequilibrium systems. As an example of a nonstationary generalized superstatistical system, I have studied the system in which the supercritical multitype Sevast'yanov process takes place. In this system, the transformation of particles of several types occurs, and the number of particles both in the whole system and in each subsystem increases exponentially. In addition, I have considered an astrophysical application of generalized superstatistics and obtained the energy distribution of ultrarelativistic electrons and positrons produced in a neutron star magnetosphere. I have found the probability that a randomly chosen particle significantly contributes to the production of secondary electron-positron pairs.

It seems interesting to find general principles that might allow us to obtain the distribution of both the intensive parameter and the control parameter. Note that in the case of ordinary superstatistics, the main approach to finding the intensive parameter distribution is maximizing either a generalized entropy or the BoltzmannGibbs-Shannon entropy under certain constraints 30 35, [57]. I believe that the same approach might be used in the case of generalized superstatistics. However, this problem requires a separate study.
[1] C. Beck and E. G. D. Cohen, Physica A 322, 267 (2003).

[2] G. Wilk and Z. Wlodarczyk, Phys. Rev. Lett. 84, 2770 (2000).

[3] C. Beck, Phys. Rev. Lett. 87, 180601 (2001).

[4] A. G. Bashkirov and A. D. Sukhanov, J. Exp. Theor. Phys. 95, 440 (2002).

[5] P. Allegrini, F. Barbi, P. Grigolini, and P. Paradisi, Phys. Rev. E 73, 046136 (2006).

[6] C. Beck, Physica A 331, 173 (2004).

[7] C. Beck, Eur. Phys. J. A 40, 267 (2009).

[8] P. Jizba and H. Kleinert, Phys. Rev. D 82, 085016 (2010).

[9] A. Y. Abul-Magd, Physica A 361, 41 (2006).

[10] A. Y. Abul-Magd, Phys. Rev. E 73, 056119 (2006).

[11] A. Y. Abul-Magd, B. Dietz, T. Friedrich, and A. Richter, Phys. Rev. E 77, 046202 (2008).

[12] S. M. Duarte Queirós, Braz. J. Phys. 38, 203 (2008).

[13] I. Lubashevsky, R. Friedrich, A. Heuer, and A. Ushakov, Physica A 388, 4535 (2009).

[14] R. F. Rodriguez and I. Santamaria-Holek, Physica A 385, 456 (2007).

[15] L. Leon Chen and C. Beck, Physica A 387, 3162 (2008).

[16] S. Abe and S. Thurner, Phys. Rev. E 72, 036102 (2005).

[17] A. Porporato, G. Vico, and P. A. Fay, Geophys. Res. Lett. 33, L15402 (2006).

[18] K. E. Daniels, C. Beck, and E. Bodenschatz, Physica D 193, 208 (2004).

[19] M. Baiesi, M. Paczuski, and A. L. Stella, Phys. Rev. Lett. 96, 051103 (2006).
[20] M. Ausloos and K. Ivanova, Phys. Rev. E 68, 046122 (2003).

[21] P. Jizba and H. Kleinert, Phys. Rev. E 78, 031122 (2008).

[22] C. Anteneodo and S. M. Duarte Queirós, J. Stat. Mech.: Theory Exp. 2009, P10023.

[23] E. Van der Straeten and C. Beck, Phys. Rev. E 80, 036108 (2009).

[24] K. Briggs and C. Beck, Physica A 378, 498 (2007).

[25] S. Rizzo and A. Rapisarda, AIP Conf. Proc. 742, 176 (2004).

[26] A. M. Reynolds, Phys. Rev. Lett. 91, 084503 (2003).

[27] S. Jung and H. L. Swinney, Phys. Rev. E 72, 026304 (2005).

[28] C. Beck, E. G. D. Cohen, and H. L. Swinney, Phys. Rev. E 72, 056133 (2005).

[29] C. Beck, Phys. Rev. Lett. 98, 064502 (2007).

[30] S. Abe, Phys. Rev. E 82, 011131 (2010).

[31] S. Abe, C. Beck, and E. G. D. Cohen, Phys. Rev. E 76, 031102 (2007).

[32] E. Van der Straeten and C. Beck, Phys. Rev. E 78, 051101 (2008).

[33] G. E. Crooks, Phys. Rev. E 75, 041119 (2007).

[34] J. Naudts, AIP Conf. Proc. 965, 84 (2007).

[35] S. Abe, Cent. Eur. J. Phys. 7, 401 (2009).

[36] F. Sattin, Eur. Phys. J. B 49, 219 (2006).

[37] B. A. Sevast'yanov, Theory Probab. Appl. 9, 521 (1964).

[38] F. R. Gantmacher, Applications of the Theory of Matrices (Interscience, New York, 1959). 
[39] V. A. Vatutin and A. M. Zubkov, J. Sov. Math. 39, 2431 (1987).

[40] B. A. Sevast'yanov and V. P. Chistyakov, Theory Probab. Appl. 16, 199 (1971).

[41] A. Yakovlev and N. Yanev, Stat. Probab. Lett. 77, 503 (2007).

[42] K. B. Athreya and N. Kaplan, Ann. Probab. 4, 38 (1976).

[43] T. Kuczek, Ann. Probab. 10, 252 (1982).

[44] M. Kramer, A. G. Lyne, J. T. O'Brien, C. A. Jordan, and D. R. Lorimer, Science 312, 549 (2006).

[45] M. A. McLaughlin, A. G. Lyne, D. R. Lorimer, M. Kramer, A. J. Faulkner, R. N. Manchester, J. M. Cordes, F. Camilo, A. Possenti, I. H. Stairs, G. Hobbs, N. D'Amico, M. Burgay, and J. T. O'Brien, Nature (London) 439, 817 (2006).

[46] Ya. N. Istomin and D. N. Sob'yanin, J. Exp. Theor. Phys. 113, 592 (2011).

[47] Ya. N. Istomin and D. N. Sob'yanin, J. Exp. Theor. Phys. 113, 605 (2011).

[48] Ya. N. Istomin and D. N. Sob'yanin, Astron. Lett. 37, 468 (2011).
[49] Ya. N. Istomin and D. N. Sob'yanin, J. Exp. Theor. Phys. 109, 393 (2009).

[50] Ya. N. Istomin and D. N. Sob'yanin, Astron. Rep. 54, 338 (2010).

[51] Ya. N. Istomin and D. N. Sob'yanin, Astron. Rep. 54, 355 (2010).

[52] Ya. N. Istomin and D. N. Sob'yanin, Astron. Lett. 33, 660 (2007).

[53] Ya. N. Istomin and D. N. Sob'yanin, AIP Conf. Proc. 983, 298 (2008).

[54] S. Asgarani and B. Mirza, Physica A 387, 6277 (2008).

[55] M. A. McLaughlin, A. G. Lyne, E. F. Keane, M. Kramer, J. J. Miller, D. R. Lorimer, R. N. Manchester, F. Camilo, and I. H. Stairs, Mon. Not. R. Astron. Soc. 400, 1431 (2009).

[56] E. F. Keane, M. Kramer, A. G. Lyne, B. W. Stappers, and M. A. McLaughlin, Mon. Not. R. Astron. Soc. 415, 3065 (2011).

[57] C. Tsallis and A. M. C. Souza, Phys. Rev. E 67, 026106 (2003). 